\title{
An automated microfluidic sample preparation system for laser scanning cytometry
}

\author{
Eric Wu • Vidya Menon • William Geddie • Yu Sun
}

Published online: 18 January 2011

(C) Springer Science+Business Media, LLC 2011

\begin{abstract}
Laser scanning cytometry (LSC) is emerging as a clinical tool. In one application a "Clatch" slide, named after the inventor, is used in conjunction with LSC for cell surface marker immunophenotyping of patient samples. The slide requires time consuming and laborious pipetting steps, making a test tedious and prone to handling errors. The Clatch slide also uses a significant number of cells, limiting the number of analyses on paucicellular samples. This paper presents an automated microfluidic system consisting of a control circuit, a microfluidic system, and an aluminum frame, capable of performing immunophenotyping procedures. This prototype system reduces 36 pipetting steps to 1 , reduces the amount of cell sample from $180 \mu \mathrm{L}$ to $56 \mu \mathrm{L}$, and shortens the time used by technicians.
\end{abstract}

Keywords Laser scanning cytometry · Microfabrication .

Microfluidic dispenser - Microfluidic connection .

Microfluidic module . Capillary burst valve $\cdot$ Passive valve

Porous membrane $\cdot$ Sample preparation $\cdot$ Automation

E. Wu $\cdot$ V. Menon $\cdot$ Y. Sun

Department of Mechanical and Industrial Engineering,

University of Toronto,

Toronto, ON M5S 3G8, Canada

E. Wu $\cdot$ Y. Sun $(\bowtie)$

Institute of Biomaterials and Biomedical Engineering,

University of Toronto,

Toronto, ON M5S 3G9, Canada

e-mail: sun@mie.utoronto.ca

W. Geddie $(\bowtie)$

Toronto General Hospital,

Toronto, ON M5G 2C4, Canada

e-mail: william.geddie@uhn.on.ca

\section{Introduction}

Quantitative Imaging Cytometry (QIC) is an emerging technology used clinically to diagnose disease and determine appropriate treatment by characterizing the antigen profile of cells (i.e., immunophenotyping). In QIC, images of adherent cells on a glass surface are used for simultaneous assessment of morphology and quantification of other parameters, usually fluorescence in multiple channels. Unlike flow cytometry, QIC allows repeated analyses of the same cells, resulting in overall lower cell number requirements for complete analysis (Gersner et al. 2006).

Laser scanning cytometry (LSC) is a form of QIC that utilizes a laser scan line ( $y$ axis) created by a mirror galvanometer and microscope stage movement ( $x$ axis) to generate images as the substrate for analysis. The scan field image is determined by the size of the microscope objective used (e.g., $398 \mu \mathrm{m}$ wide for a $20 \times$ objective, and $199 \mu \mathrm{m}$ wide for a $40 \times$ objective). LSC software contours the cells and then calculates the intensity and integral of fluorescence. These data are used to generate scattergrams and histograms used for diagnosis. A device called a "Clatch slide" (named after the inventor and shown in Fig. 1) (Clatch et al. 1998) is used for LSC testing. This device, along with the sample preparation protocol it was designed to support, is presently used by laboratories worldwide.

The Clatch slide is made of a spacer-manifold (doublesided tape template), sandwiched between a standard 1 " $\times 3$ " microscope slide and glass covers. In the standard protocol in use with this device a suspension of cells is pipetted into one end of each channel and drawn in by capillary action. The cells are allowed to settle and adhere to the glass surface by electrostatic interaction and then a pre-diluted antibody mixture is pipetted into one end of the channel while the supernatant is simultaneously absorbed at the 


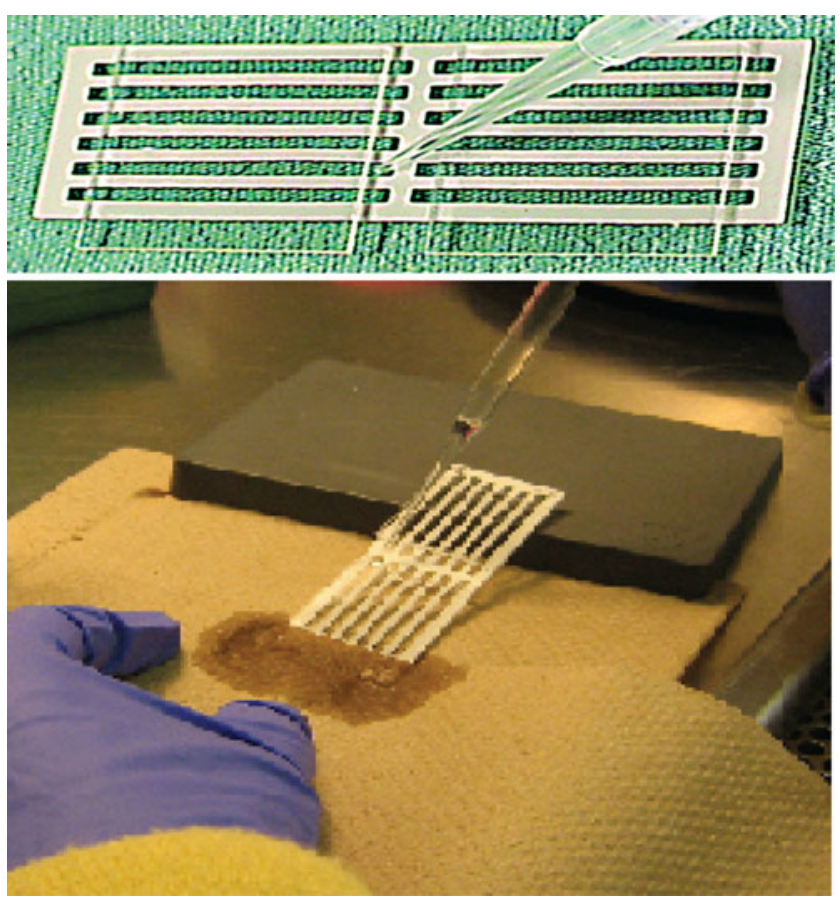

Fig. 1 Clatch slide. Top - Layout of Clatch slide. Bottom-Clatch slide reagent loading requires significant manual manipulation

other end. Accurate performance of these steps requires considerable manual dexterity and is prone to error. The size of each channel ( $2 \mathrm{~mm}$ wide, $25 \mathrm{~mm}$ long, and $200 \mu \mathrm{m}$ deep) was initially determined by considerations of ease of manual pipetting; however, the majority of cells in the well are usually not scanned because the channels of the Clatch slide, at a width of 2,000 $\mu \mathrm{m}$, and are much larger than the laser scan line $(398 \mu \mathrm{m})$. The waste of cells is significant for paucicellular sample on which a variety of investigations are required on the low number of cells available.

This paper presents a microfluidic system that improves on the Clatch slide device and procedure. The microfluidic system was developed to facilitate sample preparation for LSC. An operator loads a fluid dispenser with antibodies. The reagents in the fluid dispenser are pumped by a control circuit. The pumped reagents flow from the fluid dispenser to a disposable chip, connected by pressure contact. The disposable chip contains 12 channels allowing direct transfer of protocols used for the Clatch slide, and is scanned by the LSC. The fluid dispenser holds antibody volumes for 40 sample preparations. Passive microfluidic components were integrated into the disposable chip to control flow without requiring complicated microfluidic interconnects. The passive components include capillary burst valves, a passive valve that uses a sudden expansion of channel size and surface tension to control flow. The capillary valve has been used to control flow on a rotating disk (Chen et al. 2008). The capillary burst valve has also been previously discussed (Cho et al. 2007; Chung et al. 2005). Another passive technique used in our design is the integration of a strongly hydrophobic porous membrane to allow air to pass through, but prevent liquid from passing through.

\section{System operation}

The microfluidic system is composed of four parts: a control circuit, a fluid dispenser, a disposable chip, and a frame. Both the fluid dispenser and the disposable chip contain microfluidic channels. The control circuit contains a microcontroller (PIC18F1455) circuit, two mini-pumps (Schwarzer Precision), and 3 normally-closed minisolenoid valves (Gems Sensors). The microcontroller has a USB connection to allow the user to input custom procedures. Flexible 1/8" PVC tubing connects the control circuit to the fluid dispenser.

The frame is machined aluminum, with a recess to fit the disposable chip. A through-hole is used as a viewing port for the LSC. Figure 2 shows the fluid dispenser components and the position on the frame. A screw and spring combination on the frame is used to connect the fluid dispenser to the disposable chip. The force exerted by the spring clamp can be adjusted by turning the screws. The fluid dispenser is used to store reagents, such as antibody and rinsing agent, and dispenses them into a disposable
Fig. 2 Left-Fluid dispenser connected to disposable chip by an adjustable spring clamp. Right-Fluid dispenser components

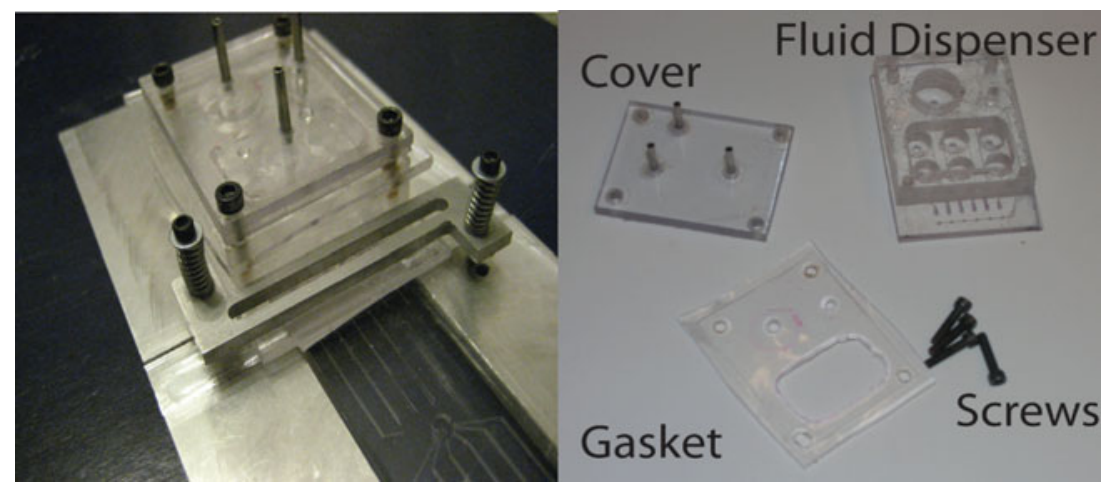


chip. The fluid dispenser contains six antibody wells, each $7 \mathrm{~mm}$ diameter and $5 \mathrm{~mm}$ high, capable of holding $192 \mu \mathrm{L}$ of antibody each. There is one larger rinsing well that is capable of holding $1.5 \mathrm{~mL}$ of rinsing agent. The amount of reagent that can be stored is equal to roughly 40 trials. A cover is screwed over the reservoirs to form an air-tight seal. Microfluidic channels connect the reservoirs to an output port that leads into the disposable chip. The microfluidic channels are used to minimize the deadvolume in the system.

The disposable chip (Fig. 3) is used as the scanned substrate in place of the Clatch slide. A center well provides the input port for the patient's cell sample, and also as the waste port for the antibody and rinsing agent during sample preparation. There are 6 channels on each side of the center well, where the cell sample settles and is scanned by the LSC. Each channel contains a vacuum port and input port. A porous membrane and capillary valve combination is used to control the flow of cell sample in the disposable chip.

Figure 4 shows the operation sequence of the system. The cell samples are first vacuumed from the center well through the vacuum ports. The cells are stopped at the vacuum port by the porous membrane, and the cells are given $15 \mathrm{~min}$ to settle and attach to the glass substrate of the disposable chip.
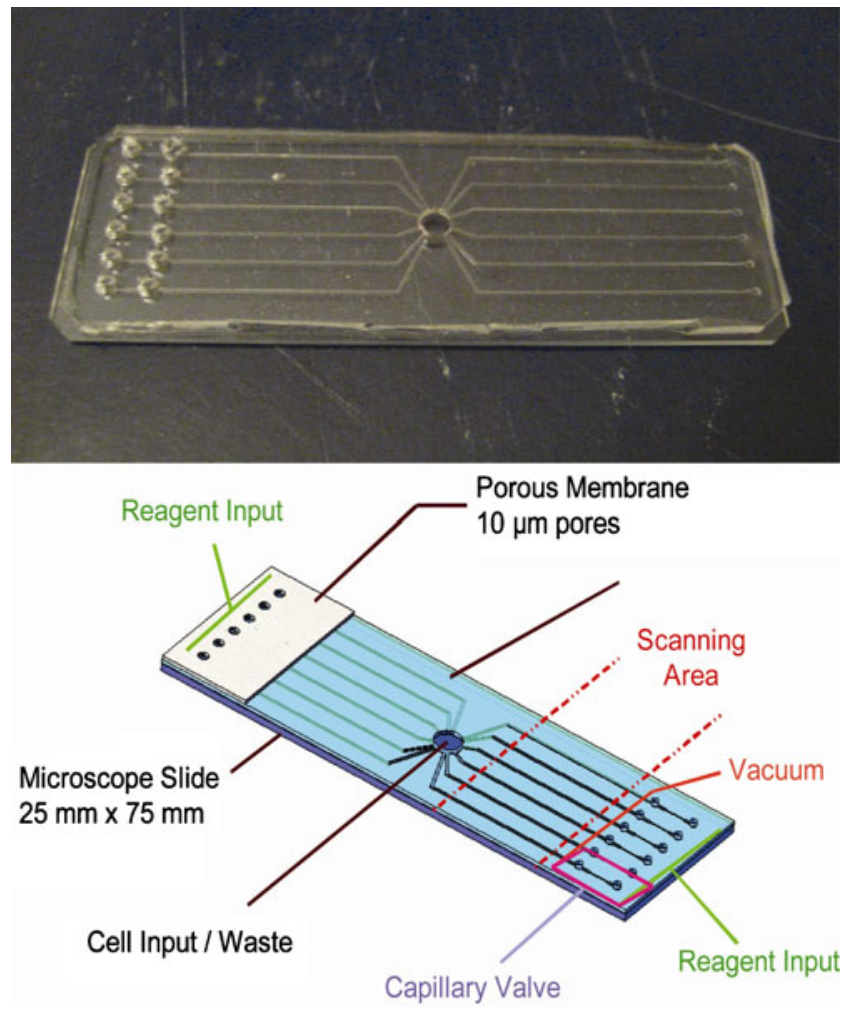

Fig. 3 Disposable chip. Top-Picture of a PDMS disposable chip bonded to a glass substrate. Bottom-disposable chip layout and description
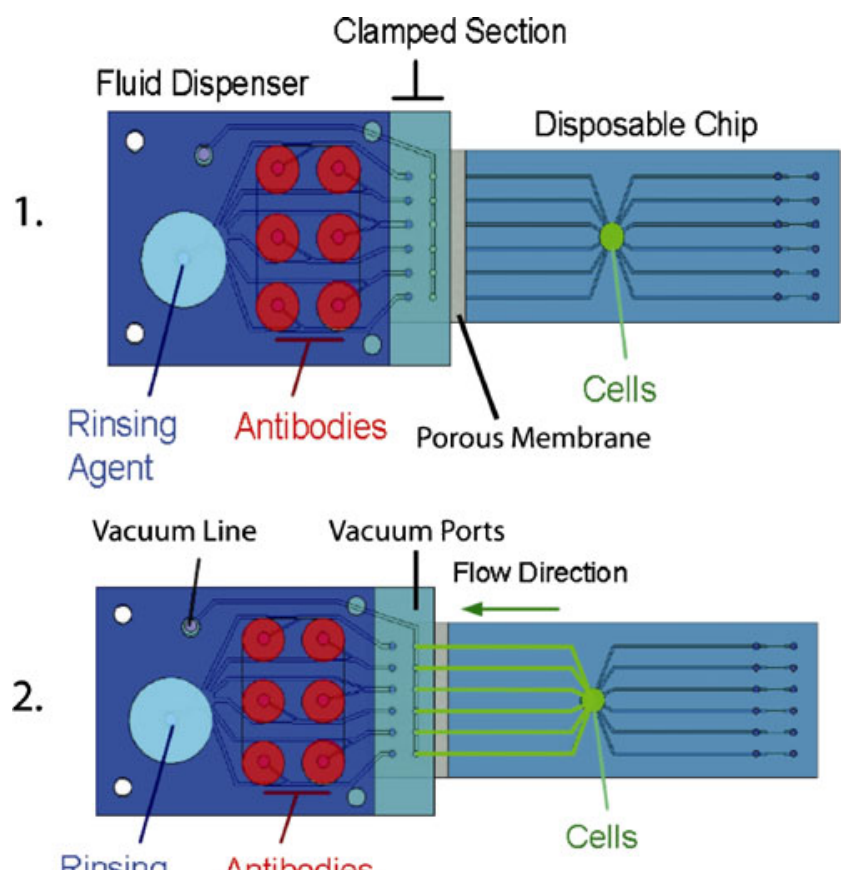

Rinsing Antibodies

Agent

Cells are brought into channels via vacuum

Flow Direction

3.

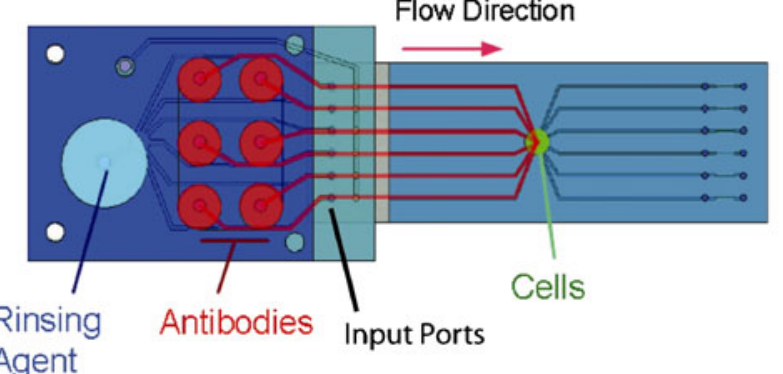

Antibody wells simultaneously pushed into channels

4.

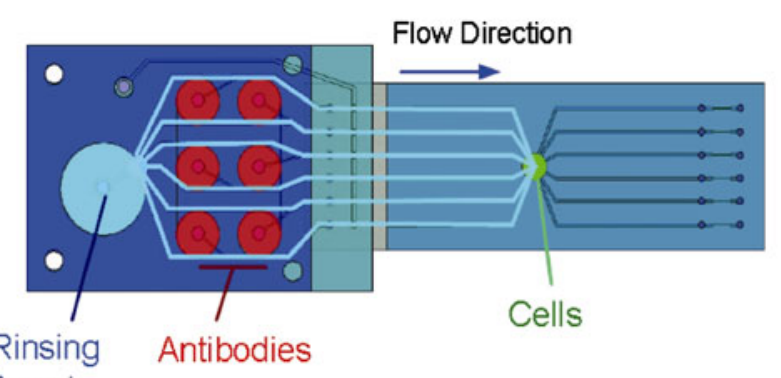

Agent

PBS rinsing agent flushes away unbound antibodies

Fig. 4 Flow order from the fluid dispenser to the disposable chip

Antibody is pumped into the channels through the input ports, and is also given $15 \mathrm{~min}$ to bond to the cell surface. Antibody solution does not block the vacuum port because the applied vacuum pressure is not sufficient to overcome the capillary burst valve. The antibodies fill the channels and exit to the center well. Once the antibodies have bound to the cells within the channels, the rinsing agent is pumped through to wash 
Fig. 5 Fluid dispenser components

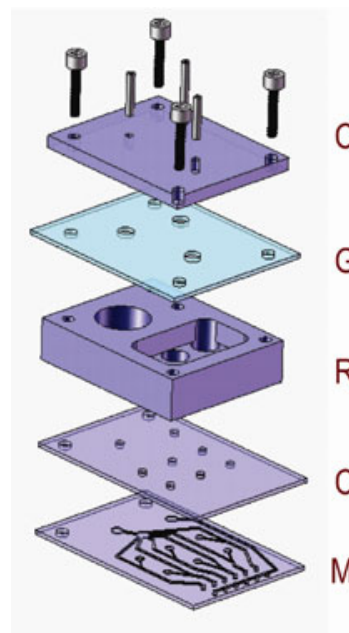

Cover

Gasket

Reservoirs

Channel Cover

Microfluidic Channels

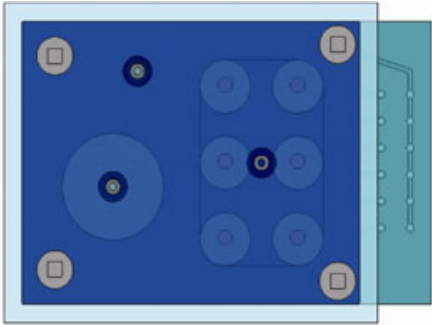

unbound antibody. The disposable chip is then scanned by the LSC.

\section{Design and fabrication}

The fluid dispenser shown in Fig. 5 is made of PMMA (RONA, Evonik) and formed by a combination of gluing and solvent-assisted hot embossing. A blank PMMA plate, $37 \mathrm{~mm} \times 46.5 \mathrm{~mm} \times 10 \mathrm{~mm}$, is drilled to create reservoirs and threaded screw holes for the cover. The reservoirs are $7 \mathrm{~mm}$ in diameter, and $5 \mathrm{~mm}$ deep, capable of holding $192 \mu \mathrm{L}$. A pocket is milled above the reservoirs that is $5 \mathrm{~mm}$ deep and serves to uniformly spread the pressure applied by the pump. The reservoirs are bonded to the microfluidic portion by a commercial solvent glue
Fig. 6 Fabrication process of copper mold
1.

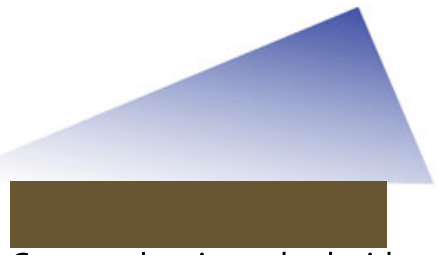

Copper plate is washed with acetone and propanol.

3.

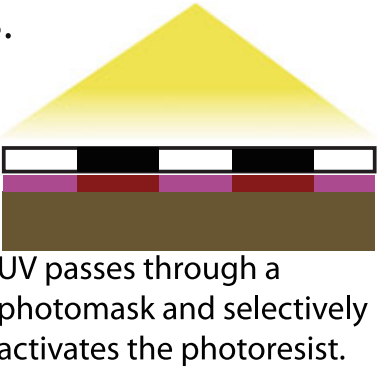

5.

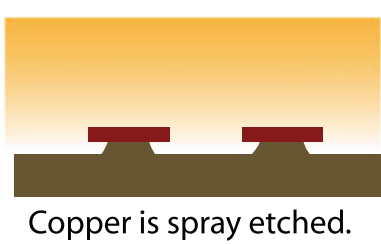

2.

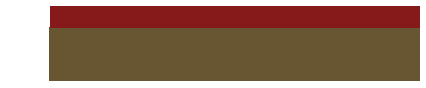

Positive photoresist is spin-coated onto the copper plate.

4.

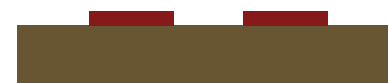

Exposed photoresist is etched away.

6.

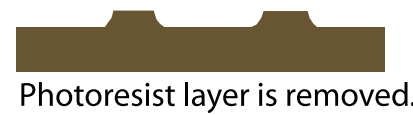


(Rez'n'Bond) made of methylene chloride. The cover, $37 \mathrm{~mm} \times 46.5 \mathrm{~mm} \times 5 \mathrm{~mm}$, is also machined PMMA and contains 3 hollow steel pins that are press-fitted and glued through, providing a connection to the rinsing reservoirs, antibody reservoir, and cell vacuum line. These pins are sized to provide an airtight seal with PVC tubing and are the connectors for the pumps and valves of the control circuit. The cover is screwed on top of the reservoirs, and a PDMS (Sylgard) gasket of 40:1 (pre-polymer:curing agent) ratio is placed between the cover and the reservoirs to form an airtight seal.

The microfluidic portion of the device is created by hot embossing a $2 \mathrm{~mm}$ thick plate of PMMA with a copper mold. Hot embossing has been extensively used for forming microfluidic devices (Tsao and Devoe 2009). Figure 6 illustrates the steps required to make our copper mold. A brushed copper plate (McMaster-Carr) is photolithographically patterned and wet etched to form a mold with microchannel features. A $10 \mu \mathrm{m}$ layer of protective positive photoresist is spincoated onto the surface of the copper plate. A photomask selectively blocks UV, and the areas exposed to UV are removed by photoresist developer. The copper plate is then wet etched in a $60^{\circ} \mathrm{C}$ ferric chloride spray etcher (Bungard). The largest channels formed were $300 \mu \mathrm{m}$ deep and $400 \mu \mathrm{m}$ wide, and the smallest were $300 \mu \mathrm{m}$ deep and $100 \mu \mathrm{m}$ wide. The etching rate was found to be approximately $20 \mu \mathrm{m} / \mathrm{min}$. After etching, the copper plate is washed vigorously.

A PMMA plate is cut to the same dimensions as the copper plate. Both items are placed into a compression molder (Carver) and are compressed together at $1.5 \mathrm{MPa}$ and heated to $135^{\circ} \mathrm{C}$ to hot emboss the copper microchannel features into the PMMA plate. The PMMA is then left to melt into the embossed shape for $1 \mathrm{~h}$. Any added pressure requires another $1 \mathrm{~h}$ wait since the melted PMMA is viscoelastic and rebounds into a slightly deformed or warped shape if it is not given time to reflow. After the $1 \mathrm{~h}$ wait time, the sample is quenched in cold water, and the

Fig. 7 Procedure flow-user and program tasks

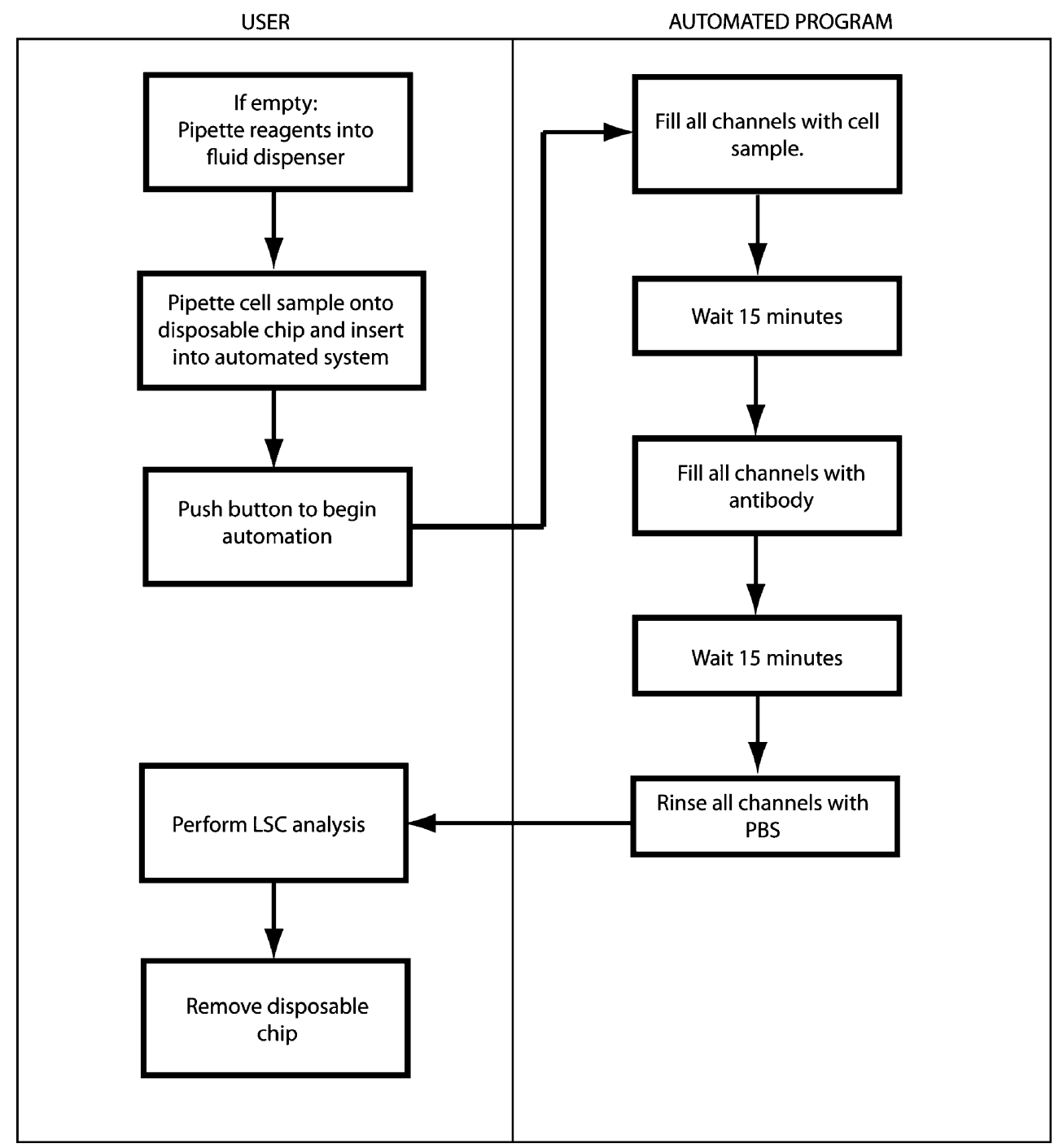


embossed plastic device is retrieved. The embossed piece is drilled to form the exit ports. A cover for the embossed part is formed by a plate with holes drilled into each of the reservoir sections. Both the cover and the embossed portion have alignment holes drilled into them that fit into a custom-made jig.

The jig is made of polycarbonate (McMaster-Carr) and steel pins, and is used to hold and align the PMMA pieces during bonding. There are several PMMA bonding techniques (Sun et al. 2007; Lin et al. 2007; Hsu and Chen 2007). The bonding technique we used is a modification of solvent-assisted thermal bonding, which was originally reported in (Klank et al. 2002). Steel plates surround the jig during bonding to maintain a uniform temperature of $87^{\circ} \mathrm{C}$. Polycarbonate has a glass transition temperature of $150^{\circ} \mathrm{C}$, and does not deform during the bonding process. An elastomeric PDMS layer (10:1 prepolymer:curing agent ratio) distributes the pressure evenly across the part to apply uniform bonding pressure. Before bonding, a $40 \%$ ethanol mixture is applied between the two plastic pieces to surface treat the plastics for greater adhesion.

The ethanol mixture (mixed by volume ratio with distilled water and $100 \%$ ethanol) allows the bonding temperature to be reduced to below the glass transition of $95^{\circ} \mathrm{C}$. This reduces the amount of deformation that occurs from pressure application. Because of the $40 \%$ dilution, the ethanol does not dissolve the material enough to block the channels. The temperature and pressure is maintained for $30 \mathrm{~min}$, and is then cooled to room temperature over another $30 \mathrm{~min}$. The pressure must be maintained during cooling; otherwise, the device will warp from the uneven temperature distribution between the top (exposed to air) and bottom (on the hot plate). After the two pieces are bonded together, the reservoirs are then glued on top of the microfluidic portion. Gluing cannot be used for bonding the microchannels because the glue would fill the channels and solidify. All microchannel features are solvent-assisted bonded, and only the macro-reservoir features are glued.

The disposable chip is made of micro-featured PDMS (polydimethyl siloxane) bonded onto a glass slide using standard soft lithography. PDMS is transparent, does not auto-fluoresce, and does not bind antibodies, making it compatible with the laser scanning cytometer. A $100 \mu \mathrm{m}$ thick layer of photoresist (SU8 Microchem) is used, and is selectively activated by UV exposure through a patterned photomask. The scanning channels are $2.2 \mathrm{~mm}$ long, $380 \mu \mathrm{m}$ wide and $100 \mu \mathrm{m}$ deep. A center well, $4 \mathrm{~mm}$ in diameter, serves as the cell suspension input. The capillary valve is a $90^{\circ}$ angle constriction, $50 \mu \mathrm{m}$ wide and $3 \mathrm{~mm}$ long. The inactivated photoresist is dissolved in a developer solution to create the mold. A PDMS ratio of 20:1 (prepolymer:curing agent) is mixed and poured over the mold. The PDMS is then baked at $80^{\circ} \mathrm{C}$ for four (4) hours, removed, hole-punched, and cut into the shape of a glass slide. The PDMS layer and a glass slide are bonded by oxygen plasma.

A porous Teflon film (Sterlitech) is placed over the disposable device vacuum ports. The membrane pores are $10 \mu \mathrm{m}$ in diameter, making the membrane gas permeable. The material is strongly hydrophobic, which prevents the cell samples from flowing past the membrane. This is necessary to stop cells from flowing into the fluid dispenser and causing contamination. The vacuum ports also serve as a bubble collector, where any bubbles originally in the device are sucked by the vacuum port. The capillary burst valve prevents reagents from the fluid dispenser from flowing into the vacuum port during the vacuuming step. The capillary valve is designed to have a burst pressure of $4.1 \mathrm{kPa}$ based on PDMS sidewalls and roof with a glass bottom (Yang and Lin 2007). The capillary valve also ensures that all the channels are filled with cell sample, regardless of varying channel lengths. The control circuit, frame, fluid dispenser, and disposable chip are assembled together to form the complete microfluidic system.

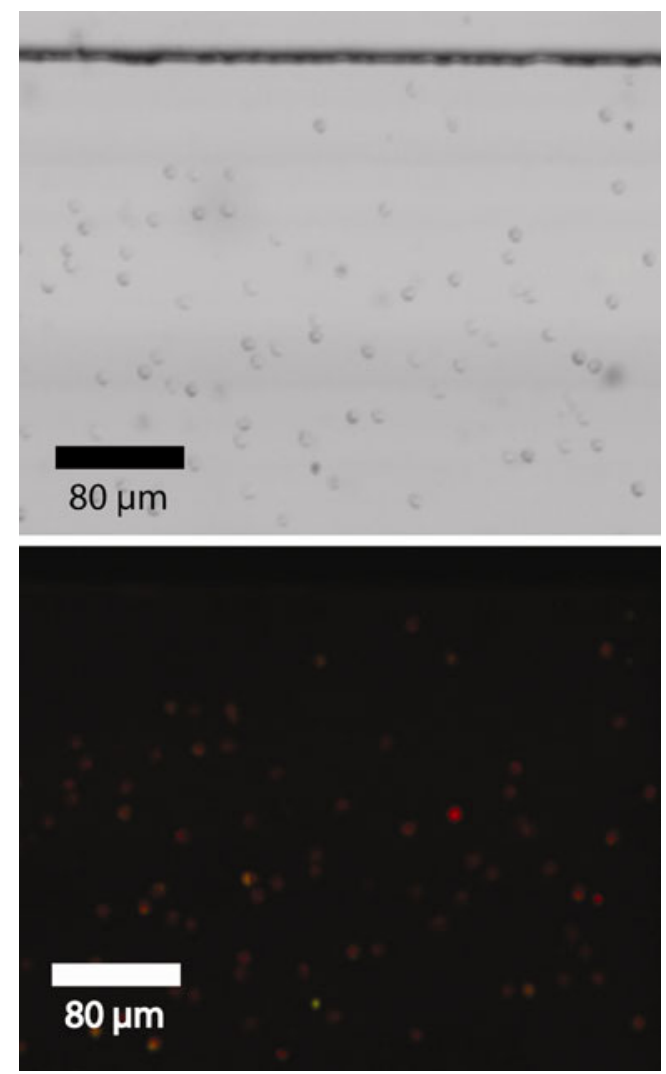

Fig. 8 LSC images of labelled cells. Cell dispersion is similar to Clatch slide, and rinsing successfully removed unbounded antibody 
Fig. 9 Scattergram comparisons between the Clatch slide and the microfluidic system producing similar results

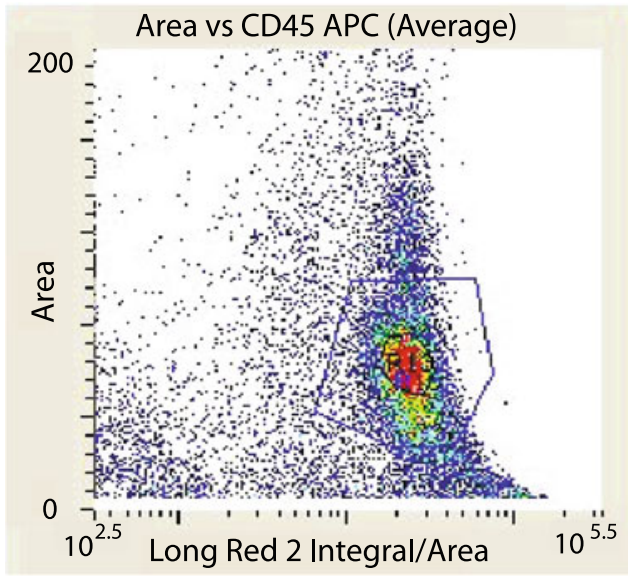

Clatch Slide
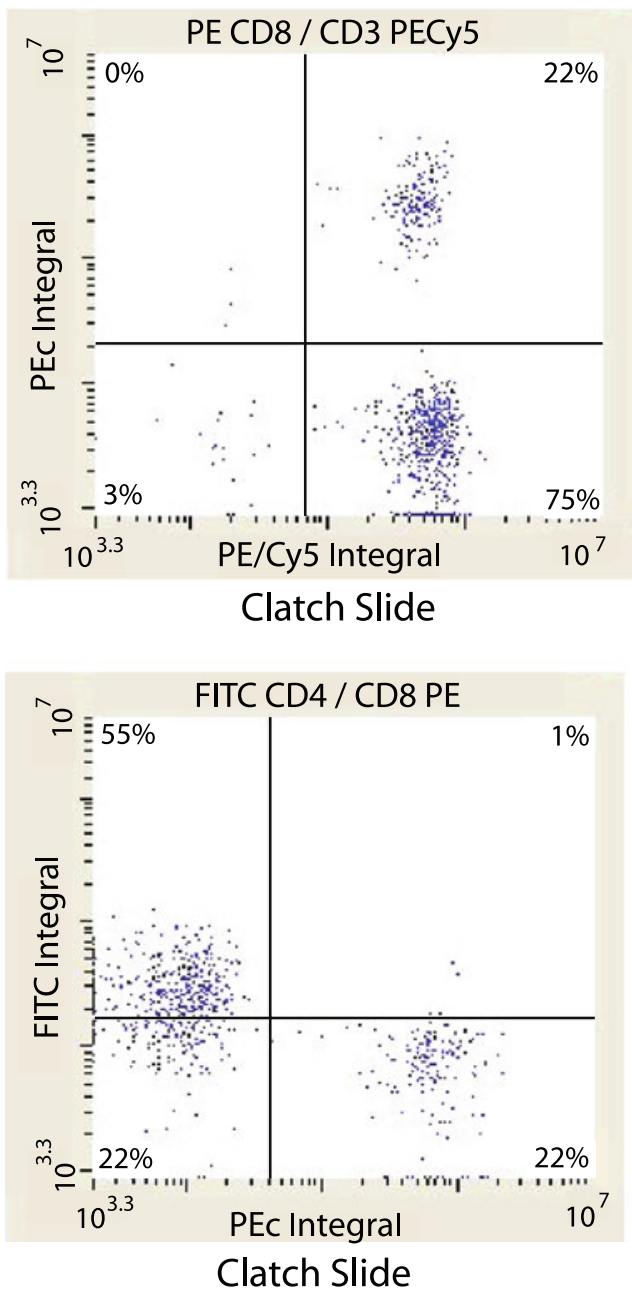
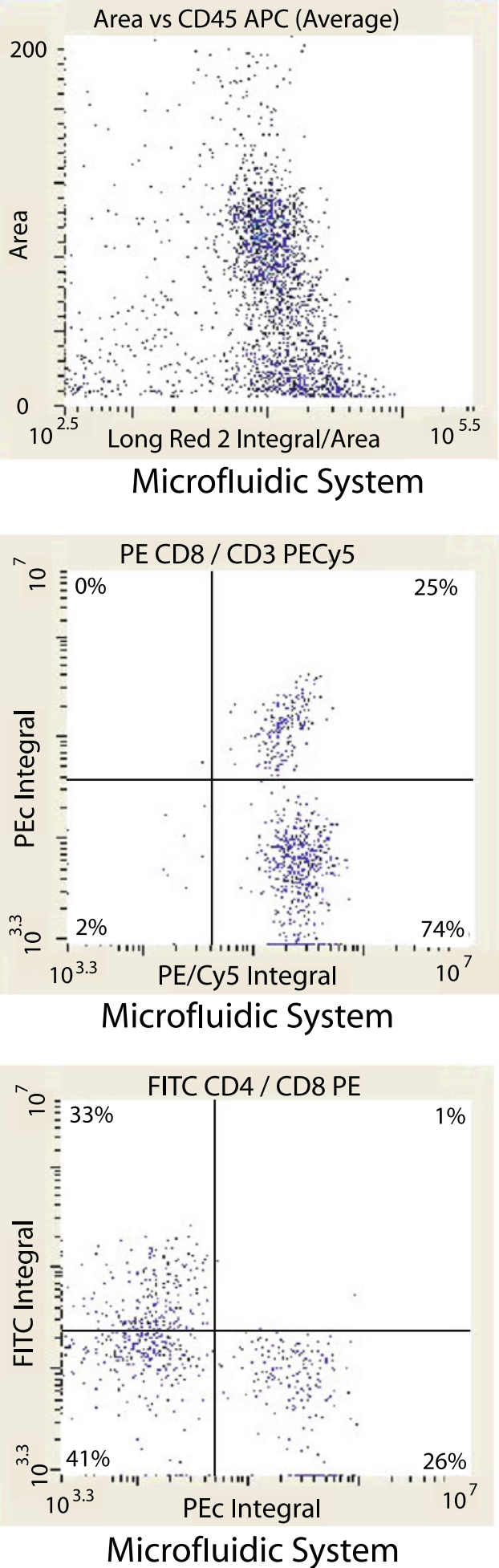

\section{Results and discussion}

Patient samples (blood lysates) were prepared by both the microfluidic system and with a Clatch slide and then analyzed by LSC. The same cell density (1-2 million cells/ $\mathrm{ml}$ ) was used for the Clatch slide and the microfluidic system. The same patient sample was used for both the Clatch slide and the microfluidic system for comparisons. Figure 7 shows the tasks for the user and the program when using the microfluidic system. Briefly, PBS and a 
Table 1 Comparison between the microfluidic system and the Clatch slide

\begin{tabular}{lll}
\hline Characteristic & Microfluidic device & Clatch slide \\
\hline 1. Cell sample volume & $56 \mu \mathrm{L}$ & $180 \mu \mathrm{L}$ \\
2. Technician time & $16 \mathrm{~s}$ & $36 \mathrm{~min}$ \\
3. Pipette steps & 1 & 36 \\
\hline
\end{tabular}

mixture of diluted fluorochrome conjugated antibodies (CD3-FITC diluted 1:20 in PBS, and CD4-PE, CD8PECy5 and CD45-APC diluted 1:10 in PBS) are pipetted into the reservoirs of the fluid dispenser. The fluid dispenser is placed on the frame and connected to an empty disposable chip. A $28 \mu \mathrm{L}$ cell suspension is pipetted into the center well of the disposable chip. When the program on the microcontroller circuit starts, a vacuum pressure is generated below the capillary valve burst pressure, filling the channels with cell suspension at a flow rate of $1.74 \mu \mathrm{L} / \mathrm{s}$. Each channel is loaded with a different set of antibodies. After antibodies have adhered to the cell surface, the channels are rinsed with PBS. It was found that a flow rate of $0.304 \mu \mathrm{L} / \mathrm{s}$ for $5 \mathrm{~s}$ in each channel was sufficient to rinse away free floating antibodies (Fig. 8). The disposable chip is then removed from the frame and placed on the LSC to gather gating scattergrams.

The Clatch slide and microfluidic system produced similar gating scattergrams (cell area vs. CD45 expression). The gating scattergrams allow the user to select the cells of interest. The clinically relevant results obtained are the same for both the microfluidic system and the Clatch slide. As shown in Fig. 9, based on the expression of CD8 vs. $\mathrm{CD} 3$, the percentage of T-suppressor cells from the leukocytes found in the blood sample was $22 \%$ for the Clatch slide, and $25 \%$ for the microfluidic system. The percentage of T-helper cells was $75 \%$ for the Clatch slide, and $74 \%$ for the microfluidic system. Based on the expression of $\mathrm{CD} 8$ vs. CD4, the percentage of $\mathrm{T}$ suppressor cells from leukocytes was found to be $22 \%$ for the Clatch slide and $26 \%$ for the microfluidic system, and the percentage of T-helper cells was found to be $77 \%$ for the Clatch slide and $74 \%$ for the microfluidic system. In clinical cytometry, differences of up to $5 \%$ in identical populations are generally considered acceptable when different aliquots of the same cell sample are tested.

The main advantages of the microfluidic system lie in less waste of cell suspension, antibody, and time in preparing a sample. Comparisons between the microfluidic system and the Clatch slide are summarized in Table 1. The Clatch slide uses approximately three times more antibody than the microfluidic device. The amount of cell suspension used was measured to be $4.6 \mu \mathrm{L}$ per channel, whereas the traditional Clatch slide used $15 \mu \mathrm{L}$ per channel. The pipetting time and steps required are also significantly reduced (36 pipetting steps down to a single pipetting step). Furthermore, the majority of cells in the channel are scanned because the width of the channel is equal to the diameter of the LSC laser, resulting in significantly less cells missed by the LSC. For the cases of misalignment between the microchannel and the reading window of the LSC, two scans were performed to double the width of the reading window. Alternatively, the width of the microchannels can be slightly increased to facilitate manufacturing tolerances.

Passive methods, such as the porous membrane and capillary burst valve successfully controlled the flow of the cell suspension, and prevented cell suspensions from entering the fluid dispenser without using expensive sensors. The Clatch slide would take approximately 6 min of manual pipetting and require the technician to return every $15 \mathrm{~min}$ between the pipetting steps. For the microfluidic system, the time required to pipette a single cell suspension and mount the disposable chip is approximately $16 \mathrm{~s}$ for each test. Additionally, after mounting the chip and starting the microfluidic system, the technician does not need to stay. The reservoirs of the fluid dispenser are much easier to pipette into, are reloadable and washable, and roughly hold enough reagent for 40 samples. The automation of the procedure effectively reduces the technician's time from 36 min to $16 \mathrm{~s}$.

\section{Conclusion}

This paper reported an automated system to perform sample preparation for LSC as an improvement over the presently used Clatch slide devices in clinical diagnostics. System design and fabrication methods were described. Passive microcomponents were proven to operate successfully in controlling fluid flow. The system demonstrated several advantages over the Clatch slide, such as less cell sample usage, less reagent usage, and faster sample preparation. Valid clinical results were demonstrated from the microfluidically prepared samples.

\section{References}

J. Chen, P. Huang, M. Lin, Microfluid. Nanofluid. 4, 427 (2008)

H. Cho, H. Kim, J. Kang, T. Kim, J. Colloid Interface Sci. 306, 379385 (2007)

K. Chung, D. Lee, H. Yang, S. Kim, H. Po, Proc. of SPIE 5651, 204 213 (2005)

R. Clatch, J. Foreman, J. Walloch, Cytometry ((Communications in Clinical Cytometry) 34, 3-16 (1998) 
A. Gersner, A. Mittag, W. Laffers, I. Dahnert, D. Lenz, F. Bootz, J. Bocsi, A. Tarnok, J. Immunol. Meth. 311, 130 128 (2006)

Y. Hsu, T. Chen, Biomed. Microdevices 9, 513-522 (2007)

H. Klank, J. Kutter, O. Geschke, Lab Chip 2, 242-246 (2002)
C. Lin, C. Chao, C. Lan, Sens. Actuators, B 121, 698-705 (2007)

X. Sun, B. Peeni, W. Yang, H. Becerril, A. Woolley, J. Chromatogr. A 1162, 162-166 (2007)

C. Tsao, D. Devoe, Microfluid. Nanofluid. 6, 1-16 (2009)

B. Yang, Q. Lin, Sens. Actuators 134, 189-193 (2007) 